\title{
SUPPORTS OF WEIGHT MODULES OVER WITT ALGEBRAS
}

\author{
VOLODYMYR MAZORCHUK AND KAIMING ZHAO
}

\begin{abstract}
In this paper, as the first step towards classification of simple weight modules with finite dimensional weight spaces over Witt algebras $W_{n}$, we explicitly describe supports of such modules. We also obtain some descriptions on the support of an arbitrary simple weight module over a $\mathbb{Z}^{n}$-graded Lie algebra $\mathfrak{g}$ having a root space decomposition $\oplus_{\alpha \in \mathbb{Z}^{n}} \mathfrak{g}_{\alpha}$ with respect to the abelian subalgebra $\mathfrak{g}_{0}$, with the property $\left[\mathfrak{g}_{\alpha}, \mathfrak{g}_{\beta}\right]=\mathfrak{g}_{\alpha+\beta}$ for all $\alpha, \beta \in \mathbb{Z}^{n}, \alpha \neq \beta$ (this class contains the algebra $W_{n}$ ).
\end{abstract}

Keywords: Witt algebra, weight module, simple module, support 2000 Math. Subj. Class.: 17B10, 17B20, 17B65, 17B66, 17B68

\section{INTRODUCTION}

Classification of simple weight modules is a classical problem in the representation theory of Lie algebras. Simple weight modules with finite-dimensional weight spaces (sometimes also called Harish-Chandra modules) are classified for several classes of algebras, including simple finite-dimensional algebras ([M2]) and various generalized Virasoro algebras ([M1, Ma3, LZ]). In the general case, however, the problem is solved only for the Lie algebra $\mathfrak{s l}_{2}$ ([Ga], see [Ma5, Chapter 3] for details).

A first step in understanding simple weight modules is to understand possible forms for the support of a module. For simple finitedimensional Lie algebras this was originated in $[\mathrm{Fe}, \mathrm{Fu}]$ and completed in $[\mathrm{DMP}]$, where it was shown that any simple weight module is either dense (that is has the maximal possible support) or is the quotient of a parabolically induced module. For some algebras of Cartan type a similar result was obtained in [PS].

A new effect appears for generalizations of the Virasoro algebra. In this case already in the class of Harish-Chandra modules there are modules whose support is one element less than the maximal possible (in what follows we call such modules punctured). Description of the support for weight modules over the Virasoro algebra is relatively easy (see $[\mathrm{Ma} 4]$ ) and for all other algebras there are only some partial results ([Ma1, Ma2]). 
The original motivation for the present paper is the problem of classification of simple Harish-Chandra modules for the Witt algebra $W_{n}$ (the algebra of derivations of a Laurent polynomial algebra in $n$ commuting variables). For the moment this problem seems to be too difficult, so as a first step in the present paper we completely determine the support of such modules, basically reducing the original classification problem to two cases: classification of simple dense modules and classification of simple punctured modules. The main result of the present paper asserts that any simple Harish-Chandra $W_{n}$-module is either dense (with uniformly bounded weight spaces) or punctured (with uniformly bounded weight spaces) or is the simple quotient of some generalized Verma module. The latter class of modules is relatively well-understood ([BZ]). It is known that both dense and punctured modules do exist ([Sh, ER]). The main result of the paper is formulated and proved in Section 3 after some preliminaries collected in Section 2.

We also obtain some partial results about the form of the support for arbitrary weight modules over $\mathbb{Z}^{n}$-graded Lie algebras $\mathfrak{g}=\bigoplus_{\alpha \in \mathbb{Z}^{n}} \mathfrak{g}_{\alpha}$ (root space decomposition with respect to the abelian subalgebra $\mathfrak{g}_{0}$ ) with the property $\left[\mathfrak{g}_{\alpha}, \mathfrak{g}_{\beta}\right]=\mathfrak{g}_{\alpha+\beta}$ for all $\alpha, \beta \in \mathbb{Z}^{n}$ with $\alpha \neq \beta$, which generalize the results in [Ma1, Ma2]. In fact, we recover and give a more detailed proof for the latter results. In particular, we show that under some mild additional assumptions the complement (in the weight lattice) to the support of a simple module is either very small (roughly speaking belongs to a sublattice of dimension $n-2$ ) or is at least a half of the lattice, see Theorem 8. This is done in Subsection 4.1. The case $n=2$ (related to [Ma1, Ma2]) is studied in details in Subsection 4.2.

We finish the paper with a brief description of similar results for socalled mixed modules. The support of a module can be refined to encode the information about finite-dimensional and infinite-dimensional weight spaces separately. A mixed module is a module which contains both finite- and infinite-dimensional weight spaces in the same coset of a weight lattice. In [MZ] it was shown that there are no simple mixed modules over the Virasoro algebra. For $W_{n}, n>1$, mixed modules do exist. However, in Subsection 4.3 we show that the part of the support of the mixed module, which describes infinite-dimensional weight spaces, behaves similarly to the support of a Harish-Chandra module. In particular, we deduce that under some mild assumptions the support of a mixed module is contained in a half of the weight lattice. We conjecture that any mixed $W_{n}$-module is neither dense nor punctured.

\section{NotATION AND PRELIMINARIES}

2.1. Weight modules over Witt algebras. We denote by $\mathbb{Z}, \mathbb{Z}_{+}, \mathbb{N}$ and $\mathbb{Q}$ the sets of all integers, nonnegative integers, positive integers and rational numbers, respectively. For any set $X$ every $\alpha \in X^{n}$ has 
the form $\alpha=\left(\alpha_{1}, \alpha_{2}, \ldots, \alpha_{n}\right)$, where $\alpha_{i} \in X$ for all $i$. For a Lie algebra $\mathfrak{a}$ we denote by $U(\mathfrak{a})$ the corresponding universal enveloping algebra.

Let $\mathbb{k}$ denote an algebraically closed field of characteristic zero. For a positive integer $n$ the corresponding classical Witt algebra $\mathfrak{W}_{n}$ is defined as the algebra of derivations of the Laurent polynomial algebra $\mathbb{k}\left[t_{1}^{ \pm 1}, \ldots, t_{n}^{ \pm 1}\right]$ in $n$ commuting variables $t_{1}, t_{2}, \ldots, t_{n}$. The algebra $\mathfrak{W}_{n}$ is simple and for $n=1$ the algebra $\mathfrak{W}_{1}$ is the centerless Virasoro algebra.

We fix a positive integer $n$ and denote $\mathfrak{g}=\mathfrak{W}_{n}$. For $i \in\{1,2, \ldots, n\}$ set $\partial_{i}=t_{i} \frac{\partial}{\partial t_{i}}$ and denote by $\mathfrak{g}_{0}$ the $\mathbb{k}$-linear span of all the $\partial_{i}{ }^{\prime}$ s. Then $\mathfrak{g}_{0}$ is an abelian Lie subalgebra of $\mathfrak{g}$, called the Cartan subalgebra.

For any $\alpha \in \mathbb{Z}^{n}$ set $t^{\alpha}=t_{1}^{\alpha_{1}} t_{2}^{\alpha_{2}} \cdots t_{n}^{\alpha_{n}}$. If $\partial \in \mathfrak{g}_{0}$ is arbitrary, then $t^{\alpha} \partial \in \mathfrak{g}$. Setting $\mathfrak{g}_{\alpha}=t^{\alpha} \mathfrak{g}_{0}$ we obtain the following decomposition of $\mathfrak{g}$ :

$$
\mathfrak{g}=\bigoplus_{\alpha \in \mathbb{Z}^{n}} \mathfrak{g}_{\alpha}
$$

It is easy to check that $\left[\mathfrak{g}_{\alpha}, \mathfrak{g}_{\beta}\right] \subset \mathfrak{g}_{\alpha+\beta}$ (and even $\left[\mathfrak{g}_{\alpha}, \mathfrak{g}_{\beta}\right]=\mathfrak{g}_{\alpha+\beta}$ unless $\alpha=\beta$ ) and hence the above decomposition, in fact, induces a $\mathbb{Z}^{n}$ grading of $\mathfrak{g}$. The adjoint action of $\mathfrak{g}_{0}$ on $\mathfrak{g}$ is diagonalizable and the decomposition (2.1) coincides with the decomposition of $\mathfrak{g}$ into a direct sum of $\mathfrak{g}_{0}$-eigenspaces.

If $\gamma \in \mathbb{k}^{n}$ is such that $\gamma_{1}, \gamma_{2}, \ldots, \gamma_{n}$ are linearly independent over $\mathbb{Q}$, then the subalgebra

$$
\operatorname{Vir}(\gamma)=\mathbb{k}\left\langle t^{\beta}\left(\gamma_{1} \partial_{1}+\gamma_{2} \partial_{2}+\cdots+\gamma_{n} \partial_{n}\right): \beta \in \mathbb{Z}^{n}\right\rangle
$$

of $\mathfrak{g}$ is a centerless generalized (or higher rank) Virasoro algebra of rank $n$ (in the sense of [PZ]). For any subgroup $G$ of $\mathbb{Z}^{n}$ there is also the corresponding subalgebra $\mathfrak{g}(G)=\bigoplus_{\alpha \in G} \mathfrak{g}_{\alpha}$ of $\mathfrak{g}$.

A $\mathfrak{g}$-module $V$ is called a weight module provided that the action of $\mathfrak{g}_{0}$ on $V$ is diagonalizable. For example, from the previous paragraph it follows that the adjoint $\mathfrak{g}$-module is a weight module. For any weight module $V$ we have the decomposition

$$
V=\bigoplus_{\lambda \in \mathfrak{g}_{0}^{*}} V_{\lambda}
$$

where $\mathfrak{g}_{0}^{*}=\operatorname{Hom}_{\mathbb{k}}\left(\mathfrak{g}_{0}, \mathbb{k}\right)$ and

$$
V_{\lambda}=\left\{v \in V: \partial v=\lambda(\partial) v \text { for all } \partial \in \mathfrak{g}_{0}\right\} .
$$

The space $V_{\lambda}$ is called the weight space corresponding to the weight $\lambda$. The support $\operatorname{supp}(V)$ of the weight module $V$ is defined as the set of all weights $\lambda$ for which $V_{\lambda} \neq 0$. If $V$ is a weight $\mathfrak{g}$-module and $\operatorname{dim}_{\mathbb{k}} V_{\lambda}<\infty$ for all $\lambda \in \mathfrak{g}_{0}^{*}$, the module $V$ is called a Harish-Chandra module.

We consider $\mathbb{Z}^{n}$ as a subset of $\mathfrak{g}_{0}^{*}$ such that each $\alpha \in \mathbb{Z}^{n}$ becomes the weight of the weight space $\mathfrak{g}_{\alpha}$ in the adjoint $\mathfrak{g}$-module (i.e. $\alpha\left(\partial_{i}\right)=$ $\alpha_{i}$ for all $\alpha \in \mathbb{Z}^{n}$ ). Under this convention, the decomposition (2.1) 
becomes a special case of the decomposition (2.2) for the adjoint $\mathfrak{g}$ module. Furthermore, if $V$ is a weight $\mathfrak{g}$-module, then for all $\alpha \in \mathbb{Z}^{n}$ and $\lambda \in \mathfrak{g}_{0}^{*}$ we have $\mathfrak{g}_{\alpha} V_{\lambda} \subset V_{\lambda+\alpha}$. From this it follows that if $V$ is an indecomposable weight module (in particular, simple), then $\operatorname{supp}(V) \subset$ $\lambda+\mathbb{Z}^{n}$ for some $\lambda \in \mathfrak{g}_{0}^{*}$.

A weight $\mathfrak{g}$-module $V$ is called

- dense provided that $\operatorname{supp}(M)=\lambda+\mathbb{Z}^{n}$ for some $\lambda \in \mathfrak{g}_{0}^{*}$;

- punctured provided that $\operatorname{supp}(M)=\mathbb{Z}^{n} \backslash\{0\}$;

- uniformly bounded provided that there exists a positive integer $N$ such that $\operatorname{dim} V_{\lambda}<N$ for all $\lambda \in \mathfrak{g}_{0}^{*}$.

2.2. Highest weight modules over Witt algebras. Choose some subgroup $G \subset \mathbb{Z}^{n}$ and some nonzero $\beta \in \mathbb{Z}^{n}$ such that $\mathbb{Z}^{n} \cong G \oplus H$, where $H$ is the subgroup of $\mathbb{Z}^{n}$, generated by $\beta$. Define the following subalgebras of $\mathfrak{g}$ :

$$
\mathfrak{a}_{G}:=\bigoplus_{\alpha \in G} \mathfrak{g}_{\alpha} ; \quad \mathfrak{n}_{G}^{+}:=\bigoplus_{\alpha \in G, k \in \mathbb{N}} \mathfrak{g}_{\alpha+k \beta} ; \quad \mathfrak{n}_{G}^{-}:=\bigoplus_{\alpha \in G, k \in \mathbb{N}} \mathfrak{g}_{\alpha-k \beta} .
$$

This gives the triangular decomposition $\mathfrak{g}=\mathfrak{n}_{G}^{-} \oplus \mathfrak{a}_{G} \oplus \mathfrak{n}_{G}^{+}$and allows us to define highest weight $\mathfrak{g}$-modules with respect to this decomposition. Note that the algebras $\mathfrak{n}_{G}^{-}$and $\mathfrak{n}_{G}^{+}$do not really depend on $\beta$ but rather on the coset $\beta+G$ (which can be chosen in two different ways, namely as $\beta+G$ or $-\beta+G)$.

Let $X$ be a simple weight $\mathfrak{a}_{G}$-module. Setting $\mathfrak{n}_{G}^{+} X=0$ we turn $X$ into a $\mathfrak{a}_{G} \oplus \mathfrak{n}_{G}^{+}$-module. We define the generalized Verma module $M(G, \beta, X)$ as follows:

$$
M(G, \beta, X):=U(\mathfrak{g}) \bigotimes_{\mathfrak{a}_{G} \oplus \mathfrak{n}_{G}^{+}} X .
$$

The module $M(G, \beta, X)$ is an indecomposable weight module and it has a unique simple quotient, which we will denote by $L(G, \beta, X)$. In [BZ] it was shown that $L(G, \beta, X)$ is a Harish-Chandra module if $X$ is a uniformly bounded exp-polynomial module. Moreover, the module $L(G, \beta, X)$ itself is not uniformly bounded unless $X$ is the trivial module (in which case $L(G, \beta, X)$ is the trivial module itself). Loosely speaking we will call $L(G, \beta, X)$ a simple highest weight module.

\section{Description of Supports For Harish-Chandra $\mathfrak{g}$-MOdules}

3.1. Formulation of the main result. Our main result is the following statement:

Theorem 1. Let $V$ be a nontrivial simple Harish-Chandra $\mathfrak{g}$-module. Then exactly one of the following statements takes place:

(a) $V$ is dense and uniformly bounded.

(b) $V$ is punctured and uniformly bounded. 
(c) $V \cong L(G, \beta, X)$ for some $G, \beta$ and a uniformly bounded $X$ as in Subsection 2.2.

For $n=1$ all Harish-Chandra modules over $\mathfrak{W}_{1}$ were classified in [M1]. In this case the statement of Theorem 1 follows immediately from this classification. Dense and punctured $\mathfrak{W}_{1}$-module occur as modules from the so-called intermediate series. In particular, in what follows we assume $n>1$.

For $n>1$ the existence of modules of the type Theorem 1(c) follows from [BZ]. Examples of both dense and punctured $\mathfrak{W}_{1}$-modules were constructed in [ER] (and in a slightly different setting already in [Sh]).

For higher rank Virasoro algebras a complete classification of all simple Harish-Chandra modules was obtained in [LZ]. From this classification it follows that the statement of Theorem 1 is true for all higher rank Virasoro algebras (simple highest weight modules over higher rank Virasoro algebras are defined exactly in the same way as in Subsection 2.2 , we will denote these modules by $L^{\mathfrak{a}}(G, \beta, X)$ where $\mathfrak{a}$ is the higher rank Virasoro algebra in question).

3.2. Auxiliary lemmata for not uniformly bounded modules. To prove Theorem 1 we will need several auxiliary statements. In the whole section we assume that $V$ is a simple Harish-Chandra $\mathfrak{g}$-module and that for some (usually fixed) $\lambda \in \mathfrak{g}_{0}^{*}$ we have $V=\oplus_{\alpha \in \mathbb{Z}^{n}} M_{\lambda+\alpha}$.

To start with, we assume that the module $V$ is not uniformly bounded. Let $e_{i}=\left(\delta_{i 1}, \delta_{i 2} \ldots, \delta_{i n}\right)$, where $\delta_{i j}$ is the Kronecker delta. Then $\left\{e_{i}: i=1,2, \ldots, n\right\}$ is the standard basis of $\mathbb{Z}^{n}$.

Lemma 2. Assume that $V$ is not uniformly bounded. Then, after an appropriate change of variables $t_{1}, t_{2}, \ldots, t_{n}$ and the weight $\lambda$, we may assume that $\lambda \neq 0$ and there exists a nonzero $v_{0} \in V_{\lambda}$ such that

$$
\mathfrak{g}_{e_{i}} v_{0}=0, \text { for all } i=1,2, \ldots, n \text {. }
$$

Proof. Choose some $\gamma \in \mathbb{k}^{n}$ such that $\gamma_{1}, \gamma_{2}, \ldots, \gamma_{n}$ are linearly independent over $\mathbb{Q}$ and consider $V$ as a Harish-Chandra $\operatorname{Vir}(\gamma)$-module, which is not uniformly bounded.

From [LZ, Theorem 3.9] and [BZ] we obtain that every uniformly bounded $\operatorname{Vir}(\gamma)$-module is either dense or punctured. Hence the total number of uniformly bounded simple subquotients of the $\operatorname{Vir}(\gamma)$ module $V$ is finite (it is bounded by $\operatorname{dim}_{\mathbb{k}} V_{0}+\operatorname{dim}_{\mathbb{k}} V_{\mu}<\infty$ for any nonzero $\mu \in \lambda+\mathbb{Z}^{n}$ ). As the module $V$ itself is not uniformly bounded, it must contain a simple $\operatorname{Vir}(\gamma)$-subquotient, say $X$, which is not uniformly bounded. By [LZ, Theorem 3.9], the module $X$ is a highest weight $\operatorname{Vir}(\gamma)$-module and, after an appropriate change of variables $t_{1}, t_{2}, \ldots, t_{n}$ and the weight $\lambda$, the module $X$ is isomorphic to the module $L^{\operatorname{Vir}(\gamma)}\left(G, e_{1}, Y\right)$, where $G=\mathbb{Z}\left\langle e_{2}, e_{3}, \ldots, e_{n}\right\rangle$ and $Y$ is the corresponding simple uniformly bounded $\mathfrak{b}_{G}$-module (here $\mathfrak{b}_{G}=\mathfrak{a}_{G} \cap \operatorname{Vir}(\gamma)$ ). 
By [BZ] we have that the dimensions $\operatorname{dim}_{\mathbb{k}} V_{-k e_{1}+\lambda}, k \in \mathbb{N}$, are not uniformly bounded.

Fix an integer $N>3$ and consider the finite set

$$
B_{N}(\lambda)=\lambda+\left\{\alpha \in \mathbb{Z}^{n}:\left|\alpha_{i}\right| \leqslant N \text { for all } i\right\} .
$$

As $V$ is a Harish-Chandra module and $\operatorname{dim}_{\mathbb{k}} V_{-k e_{1}+\lambda}, k \in \mathbb{N}$, are not uniformly bounded, there exists a positive integer $k$ such that we have $-k e_{1}+\lambda \neq 0$ and

$$
\operatorname{dim}_{\mathbb{k}} V_{-k e_{1}+\lambda}>n \sum_{\beta \in B_{N}(\lambda)} \operatorname{dim}_{\mathbb{k}} V_{\beta} .
$$

Set $e_{1}^{\prime}=(k+1) e_{1}+e_{2}, e_{2}^{\prime}=k e_{1}+e_{2}$, and, finally, $e_{j}^{\prime}=e_{1}^{\prime}+e_{j}$ for all $j=3,4, \ldots, n$. Then $\left\{e_{1}^{\prime}, e_{2}^{\prime}, \ldots, e_{n}^{\prime}\right\}$ is a new $\mathbb{Z}$-basis of $\mathbb{Z}^{n}$. Moreover, for any $i=1,2, \ldots, n$ we have $e_{i}^{\prime}+\left(-k e_{1}+\lambda\right) \in B_{N}(\lambda)$. Note that $\operatorname{dim}_{\mathbb{k}} \mathfrak{g}_{e_{i}^{\prime}}=n$ (observe the factor $n$ in the right hand side of (3.2)). Hence, because of our choice of $k$ above, there exists a nonzero $v_{0} \in V_{-k e_{1}+\lambda}$ such that $\mathfrak{g}_{e_{i}^{\prime}} v_{0}=0$ for all $i$. Thus, after another change of variables (corresponding to the choice of the $\mathbb{Z}$-basis $\left\{e_{i}^{\prime}\right\}$ of $\mathbb{Z}^{n}$ ) and replacement of $\lambda$ with $-k e_{1}+\lambda$, we obtain $v_{0} \in V_{\lambda}$ such that $\mathfrak{g}_{e_{i}} v_{0}=0$ for all $i=1,2, \ldots, n$. This completes the proof of the lemma.

To proceed we need some more notation. For any $\alpha, \beta \in \mathbb{Z}^{n}$, we write $\alpha>\beta$ and $\alpha \geqslant \beta$ if $\alpha_{i}>\beta_{i}$ or $\alpha_{i} \geqslant \beta_{i}$ for $i=1,2, \ldots, n$, respectively. For $p, q \in \mathbb{Z}$ we set $[p, q]=\{x \in \mathbb{Z}: p \leqslant x \leqslant q\}$ and define $(-\infty, q]$ and $[p,+\infty)$ similarly.

An element $v \in V$ will be called a generalized highest weight element provided that there exists some $N \in \mathbb{N}$ such that $\mathfrak{g}_{\alpha} v=0$ for every $\alpha \in \mathbb{Z}^{n}$ such that $\alpha>(N, N, \ldots, N)$. Analogues for different algebras of the next two claims appeared in various disguises and setups e.g. in [Ma1, Ma2, Su, LZ].

Lemma 3. Let $X$ be a weight $\mathfrak{g}$-module and $x \in X$ be a generalized highest weight element. Then every element in $U(\mathfrak{g}) x$ is a generalized highest weight element. In particular, if $V$ is as in Lemma 2 satisfying (3.1), then every $v \in V$ is a generalized highest weight element.

Proof. Since the algebra $U(\mathfrak{g})$ is generated by all $\mathfrak{g}_{\alpha}$, to prove the first assertion it is enough to show that if $x$ is a generalized highest weight element, $\beta \in \mathbb{Z}^{n}$ and $a \in \mathfrak{g}_{\beta}$, then the element $a x$ is a generalized highest weight element.

Assume that $N \in \mathbb{N}$ is such that $\mathfrak{g}_{\alpha} v=0$ for every $\alpha \in \mathbb{Z}^{n}$ with $\alpha>(N, N, \ldots, N)$. Set $N^{\prime}=N+\left|\beta_{1}\right|+\left|\beta_{2}\right|+\cdots+\left|\beta_{n}\right|+1$. Then for every $\alpha>\left(N^{\prime}, N^{\prime}, \ldots, N^{\prime}\right)$ we have

$$
\mathfrak{g}_{\alpha} \mathfrak{g}_{\beta} v \subset \mathfrak{g}_{\beta} \mathfrak{g}_{\alpha} v+\mathfrak{g}_{\beta+\alpha} v=0
$$

as $\alpha, \beta+\alpha>(N, N, \ldots, N)$ by our choice of $N^{\prime}$. The first assertion of the lemma follows. 
The module $V$ from Lemma 2 is simple and hence is generated by every nonzero element. By (3.1), the element $v_{0}$ is a generalized highest weight element. Therefore the second assertion of our lemma follows directly from the first assertion.

Lemma 4. Let $V$ be as in Lemma 2 satisfying (3.1). Then $\mathfrak{g}_{-\alpha} v \neq 0$ for any nonzero $v \neq V$ and any $\alpha \in \mathbb{N}^{n}$.

Proof. Assume that $\mathfrak{g}_{-\alpha} v=0$ for some nonzero $v \neq V$. By Lemma 3, there exists $N \in \mathbb{N}$ such that $\mathfrak{g}_{e_{i}+N \alpha} v=0$ for every $i=1,2, \ldots, n$. It is easy to see that the monoid $\mathbb{Z}^{n}$ is generated, as a monoid, by the elements $e_{i}+N \alpha, i=1,2, \ldots, n$, and $-\alpha$. It follows that $\mathfrak{W}_{n}$ is generated, as a Lie algebra, by $\mathfrak{g}_{e_{i}+N \alpha}, i=1,2, \ldots, n$, and $\mathfrak{g}_{-\alpha}$. Hence $\mathfrak{W}_{n} v=0$, which implies that $V$ is the trivial module. This contradicts our assumption that $V$ is not uniformly bounded and the claim of the lemma follows.

Analogues of the next two claims appeared in the setup of generalized Virasoro algebras in [LZ, Lemma 3.1]. Our proofs below are generalizations of the ones from [LZ].

Lemma 5. Let $V$ be as in Lemma 2 satisfying (3.1). Then for any $\mu \in \operatorname{Supp}(V)$ and any $\alpha \in \mathbb{N}^{n}$ we have

$$
\{x \in \mathbb{Z}: \mu+x \alpha \in \operatorname{Supp}(V)\}=(-\infty, m]
$$

for some $m \in \mathbb{N} \cup\{0\}$.

Proof. Set $J:=\{x \in \mathbb{Z}: \mu+x \alpha \in \operatorname{Supp}(V)\}$. From Lemma 4 we have that either $J=(-\infty, m]$ for some $m \in \mathbb{Z}$ or $J=\mathbb{Z}$. Suppose that $J=\mathbb{Z}$. Choose some $\partial \in \mathfrak{g}_{0}$ such that $\partial\left(t^{\beta}\right)=0$ implies $\beta=0$ for all $\beta \in \mathbb{Z}^{n}$.

Consider the subalgebra $\mathfrak{V}_{\alpha}$ of $\mathfrak{g}$, generated by the elements $t^{k \alpha} \partial$, $k \in \mathbb{Z}$. This subalgebra is a classical centerless Virasoro algebra (of rank one) and the space $X_{\alpha}:=\oplus_{x \in \mathbb{Z}} V_{\mu+x \alpha}$ admits a natural structure of a $\mathfrak{V}_{\alpha}$-module, given by restriction.

From Lemma 3 we obtain that for any $v \in X_{\alpha}$ we have $t^{k \alpha} \partial(v)=0$ for all $k \in \mathbb{N}$ big enough. Hence, by [M1, Lemma 1.6], for every $m \in \mathbb{Z}$ there exists $m^{\prime} \in \mathbb{Z}, m^{\prime}>m$, and a nonzero vector $v\left(m^{\prime}\right) \in V_{\mu+m^{\prime} \alpha}$ annihilated by $t^{k \alpha} \partial$ for all $k \in \mathbb{N}$.

Therefore the weight $\mu$ occurs in infinitely many simple highest weight $\mathfrak{V}_{\alpha}$-subquotients of $X_{\alpha}$, implying $\operatorname{dim}_{\mathbb{k}} V_{\mu}=\infty$. This contradicts our assumption that $V$ is a Harish-Chandra module. Thus $J \neq \mathbb{Z}$ and the claim of the lemma follows.

Lemma 6. Let $V$ be as in Lemma 2 satisfying (3.1). Then one can change the variables $t_{1}, t_{2}, \ldots, t_{n}$ (keeping the weight $\lambda$ ) such that there exists $v_{0} \in V_{\lambda}$ with the following properties:

(a) The condition (3.1) is satisfied. 
(b) $\lambda+\alpha \notin \operatorname{supp}(V)$ for any nonzero $\alpha \in \mathbb{Z}_{+}^{n}$.

(c) $\lambda-\alpha \in \operatorname{supp}(V)$ for any $\alpha \in \mathbb{Z}_{+}^{n}$.

(d) for any $\alpha, \beta \in \mathbb{Z}^{n}$ such that $\alpha \leqslant \beta$ we have $\lambda+\alpha \notin \operatorname{supp}(V)$ implies $\lambda+\beta \notin \operatorname{supp}(V)$.

Proof. By Lemma 5 we have $\{x \in \mathbb{Z}: \lambda+x(1,1 \cdots, 1) \in \operatorname{supp}(V)\}=$ $(\infty, p-2]$ for some $p \geqslant 2$. Take $e_{1}^{\prime}=(p+1, p, \ldots, p), e_{2}^{\prime}=(p+2, p+$ $1, p, \ldots, p)$ and $e_{i}^{\prime}=e_{1}^{\prime}+e_{i}$ for all $i=3,4, \ldots, n$. This gives us a new $\mathbb{Z}$-basis of $\mathbb{Z}^{n}$. The condition (a) is obvious. The condition (d) follows from Lemma 5. The conditions (b) and (c) are proved similarly to the proof of Lemma 4 (note that $\lambda+(p-1)(1,1, \ldots, 1) \notin \operatorname{supp}(V)$ ).

3.3. The key lemma. The following statement is our key observation. For $a, b \in \mathbb{Z}^{n}$, we set $a \cdot b=a_{1} b_{1}+a_{1} b_{1}+\ldots+a_{1} b_{1}$. For any subgroup $G$ of $\mathbb{Z}^{n}$ and any $\mu \in \mathfrak{g}_{0}^{*}$ the space $V(\mu+G)=\bigoplus_{\alpha \in \mu+G} V_{\alpha}$ is naturally a $\mathfrak{g}(G)$-module by restriction.

Lemma 7. Assume that $V$ is not uniformly bounded. Then $V$ is a highest weight module as in Theorem 1(c).

Proof. By Lemma 6 we may assume that $V$ satisfies Lemma 6(a)-(d).

Fix $\gamma \in \mathbb{k}^{n}$ such that $\gamma_{1}, \gamma_{2}, \cdots, \gamma_{n}$ are linearly independent over $\mathbb{Q}$ and consider $V$ as a Harish-Chandra $\operatorname{Vir}(\gamma)$-module by restriction. To simplify our notation, set $\mathfrak{a}:=\operatorname{Vir}(\gamma)$. Let $Y$ be a minimal $\mathfrak{a}$-submodule of $V$ such that $Y \cap V_{\lambda} \neq 0$, and $Z$ the maximal a-submodule of $Y$ such that $Z \cap V_{\lambda}=0$. Then the $\mathfrak{a}$-module $Y / Z$ is simple.

As both $\lambda \neq 0$ and $V_{\lambda} \neq 0$ by Lemma 6 , from [LZ, Theorem 3.9] it follows that the a-module $Y / Z$ is isomorphic to $L^{\mathfrak{a}}(G, \beta, X)$ for some $G, \beta$ and $X$ as described in Subsection 2.2 (but for the algebra $\mathfrak{a}$ and after the identification of $\lambda$ with $\left.\lambda\left(\gamma_{1} \partial_{1}+\gamma_{2} \partial_{2}+\cdots+\gamma_{n} \partial_{n}\right)\right)$. Moreover, $X$ is uniformly bounded (and hence is a module from the intermediate series). It now follows that

$$
\left(\lambda-\mathbb{Z}_{+} \beta+G\right) \backslash\{0\} \subset \operatorname{Supp}(V) .
$$

Moreover, from Lemma 6(b) we have $\lambda+\alpha+G \not \subset \operatorname{Supp}(V)$ for any nonzero $\alpha \in \mathbb{Z}_{+}^{n}$.

From Lemma 6(b) it follows that there exists $\alpha \in \mathbb{N}^{n}$ such that we have $G=\left\{x \in \mathbb{Z}^{n}: \alpha \cdot x=0\right\}$. (For example, if $\alpha_{1}=0$, then $l e_{1} \in G$ for all $l \in \mathbb{Z}$, and $\lambda+l_{1} e_{1} \notin \operatorname{supp}(V)$ for sufficiently large $l_{1}$ which contradicts the fact that $(\lambda+G) \backslash\{0\} \subset \operatorname{Supp}(V)$.) It follows from (3.3) that $\lambda+x \in \operatorname{Supp}(V)$ for all $x \in \mathbb{Z}^{n}$ with $x \cdot \alpha<0$.

We first consider the case when $\{\lambda+k \beta+G\} \cap \operatorname{supp}(V)=\varnothing$ for some $k \in \mathbb{N}$. We may assume that $k$ is minimal possible. In this case for any $\mu \in\{\lambda+(k-1) \beta+G\} \cap \operatorname{supp}(V)$ (note that the latter intersection is not empty because of our assumption on $k$ ) and any $x \in G$ we have 
$\mathfrak{g}_{x+\beta} V_{\mu}=0$. Hence $V \cong L\left(G, \beta, X^{\prime}\right)$, where

$$
X^{\prime}=\bigoplus_{\mu \in\{\lambda+(k-1) \beta+G\}} V_{\mu}
$$

Now consider the remaining case when $\{\lambda+k \beta+G\} \cap \operatorname{supp}(V) \neq \varnothing$ for all $k \in \mathbb{N}$. Obviously, we can choose $k \in \mathbb{N}$ big enough such that the following two conditions are satisfied:

(I) $\left|\{\lambda+k \beta+G\} \cap\left\{\lambda+\mathbb{Z}_{+}^{n}\right\}\right|>1$

(II) $\left|\{\lambda+(k-1) \beta+G\} \cap\left\{\lambda+\mathbb{Z}_{+}^{n}\right\}\right|>0$.

The space $V(\lambda+k \beta+G)$ is a Harish-Chandra $\mathfrak{g}(G)$-module. Restricting this module to any generalized Virasoro subalgebra of $\mathfrak{g}(G)$ (of the same rank) and using (I), [LZ] and [BZ] in the same way as we did in the proof of Lemma 2, we get that this module is not uniformly bounded. Hence we can repeat the arguments of Lemma 2 and find a $\mathbb{Z}$-basis $\beta_{2}, \beta_{3}, \ldots, \beta_{n}$ of $G, \mu \in \lambda+k \beta+G$, and a nonzero element $v \in V_{\mu}$, such that $\mathfrak{g}_{\beta_{i}} v=0$ for all $i=2,3, \ldots, n$.

Let $\nu \in\{\lambda+(k-1) \beta+G\} \cap\left\{\lambda+\mathbb{Z}_{+}^{n}\right\}$, which exists by (II). Then $\nu \notin \operatorname{supp}(V)$ by Lemma $6(\mathrm{~b})$. Let $\beta_{1}^{\prime}=\nu-\mu$. Then $\beta_{1}^{\prime}, \beta_{2}, \beta_{3}, \ldots, \beta_{n}$ is a $\mathbb{Z}$-basis of $\mathbb{Z}^{n}$ and $\mathfrak{g}_{\beta_{1}^{\prime}} v=0$ as well.

From Lemma 5 we obtain that $\mu+m\left(\beta_{1}^{\prime}+\beta_{2}+\ldots+\beta_{n}\right) \notin \operatorname{supp}(V)$ for all sufficiently large integers $m$. At the same time for $m \in \mathbb{N}$ we have

$$
\alpha \cdot m\left(\beta_{1}^{\prime}+\beta_{2}+\ldots+\beta_{n}\right)=m \alpha \cdot \beta_{1}^{\prime}=-m \alpha \cdot \beta<0 .
$$

Hence for all $m$ sufficiently large we have

$$
\mu+m\left(\beta_{1}^{\prime}+\beta_{2}+\ldots+\beta_{n}\right) \in \lambda-\mathbb{Z}_{+} \beta+G
$$

This contradicts (3.3). Hence it is not possible that the intersection $\{\lambda+k \beta+G\} \cap \operatorname{supp}(V)$ is nonempty for all $k \in \mathbb{N}$. The claim of the lemma follows.

3.4. Proof of Theorem 1. From Lemma 5 it follows that every simple dense and every simple punctured $\mathfrak{g}$-module is uniformly bounded. Let now $V$ be a simple nontrivial Harish-Chandra $\mathfrak{g}$-module, which is neither dense nor punctured. Let $\gamma \in \mathbb{k}^{n}$ be such that $\gamma_{1}, \gamma_{2}, \ldots, \gamma_{n}$ are linearly independent over $\mathbb{Q}$. Then $V$ is a $\operatorname{Vir}(\gamma)$-module by restriction. As $V$ is neither dense nor punctured, from [LZ, Theorem 3.9] it follows that every simple nontrivial subquotient of $V$ is a highest weight module. In particular, $V$ is not uniformly bounded by [BZ]. From Lemma 7 we now get that $V$ is a highest weight $\mathfrak{g}$-module as described in Theorem 1(c). This completes the proof. 


\section{Support of NON HARISh-Chandra Weight MOdules}

In this section we would like to prove some analogue of Theorem 1 for all weight modules (that is without the assumption to be a HarishChandra module). At this moment, we cannot get such a nice statement as the one in Theorem 1, but we get some information about the support of the module generalizing the corresponding result for the Virasoro algebra ([Ma4, Theorem 2]).

Actually in this section our algebra $\mathfrak{g}$ can be more general than $W_{n}$. We assume that $\mathfrak{g}$ has a $\mathbb{Z}^{n}$-gradation $\mathfrak{g}=\bigoplus_{\alpha \in \mathbb{Z}^{n}} \mathfrak{g}_{\alpha}$ such that $\mathfrak{g}_{0}$ is abelian and the gradation itself is the root space decomposition with respect to $\mathfrak{g}_{0}$. We also assume that $\left[\mathfrak{g}_{\alpha}, \mathfrak{g}_{\beta}\right]=\mathfrak{g}_{\alpha+\beta}$ for all $\alpha, \beta \in \mathbb{Z}^{n}$ with $\alpha \neq \beta$. Clearly both $W_{n}$ and higher rank Virasoro algebras are examples of such Lie algebras.

4.1. Cut modules. For $a \in \mathbb{R}^{n}$ set

$$
\begin{aligned}
& \mathbb{Z}_{-}^{(a)}=\left\{x \in \mathbb{Z}^{n}: a \cdot x<0\right\} \\
& \mathbb{Z}_{+}^{(a)}=\left\{x \in \mathbb{Z}^{n}: a \cdot x>0\right\} ; \\
& \mathbb{Z}_{0}^{(a)}=\left\{x \in \mathbb{Z}^{n}: a \cdot x=0\right\} ; \\
& \mathbb{Z}_{-0}^{(a)}=\left\{x \in \mathbb{Z}^{n}: a \cdot x \leqslant 0\right\} ;
\end{aligned}
$$

Following [Ma1, Ma2] we call a simple weight $\mathfrak{g}$-module $V$ cut provided that there exists $\lambda \in \operatorname{supp}(V), a \in \mathbb{R}^{n}, a \neq 0$, and $b \in \mathbb{Z}^{n}$ such that $\operatorname{supp}(V) \subset \lambda+b+\mathbb{Z}_{-0}^{(a)}$.

Let $L(G, \beta, X)$ be a simple highest weight module as in Subsection 2.2. It is easy to see that there exists $a \in \mathbb{R}^{n}, a \neq 0$, such that $G \subset \mathbb{Z}_{0}^{(a)}$ and $\beta \in \mathbb{Z}_{+}^{(a)}$ (actually in this case we can even take $\left.a \in \mathbb{Z}^{n}\right)$. For any $\lambda \in \operatorname{supp}(X)$ we have $\operatorname{supp}(L(G, \beta, X)) \subset \lambda+\mathbb{Z}_{-0}^{(a)}$, in particular, $L(G, \beta, X)$ is a cut module (where we take $b=0$ ).

In the general case one cannot get rid of the element $b$ in the definition of cut modules. Choose some $a \in \mathbb{R}^{n}, a \neq 0$, such that $\mathbb{Z}_{0}^{(a)}=\{0\}$. Set

$$
\mathfrak{n}^{ \pm}=\bigoplus_{\alpha \in \mathbb{Z}_{ \pm}^{(a)}} \mathfrak{g}_{\alpha}
$$

We make $\mathbb{k}$ as the trivial $\mathfrak{b}=\mathfrak{g}_{0} \oplus \mathfrak{n}^{+}$-module. Then the kernel of the canonical epimorphism from the Verma module $U(\mathfrak{g}) \otimes_{U(\mathfrak{b})} \mathbb{k}$ onto the trivial $\mathfrak{g}$-module contains a weight irreducible subquotient with support $\mathbb{Z}_{-}^{(a)}$ since $\mathfrak{g}$ contains a rank $n$ Virasoro algebra(using [HWZ]).

Our main result of this subsection is the following: 
Theorem 8. Let $V$ be a simple weight $\mathfrak{g}$-module, which is neither dense nor trivial. Assume that $V$ contains a generalized highest weight element for some choice of variables $t_{1}, \ldots, t_{n}$. Then $V$ is a cut module.

Proof. For $n=1$ this is proved in [Ma4, Theorem 2], so in what follows we assume $n>1$. By Lemma 3, every element of $V$ is a generalized highest weight element with respect to our fixed choice of $t_{1}, \ldots, t_{n}$. We will use real convexity theory in our arguments, so we will need to fix the corresponding setup.

Fix $\lambda \in \operatorname{supp}(V), \lambda \neq 0$, and consider the set $\Lambda=\lambda+\mathbb{Z}^{n}$, which we identify with $\mathbb{Z}^{n}$ and consider as a subset of $\mathbb{R}^{n}$. Set further $\hat{\Lambda}=$ $\Lambda \backslash \operatorname{supp}(V)$ and note that $\hat{\Lambda} \neq \varnothing$ because of our assumption that $V$ is not dense. The key point of the proof is the following observation, which establishes a convexity property for $\hat{\Lambda}$ :

Lemma 9. Let $\mu \in \Lambda$ be a convex linear combination of some elements from $\hat{\Lambda}$. Then either $\mu=0$ or $\mu \in \hat{\Lambda}$.

Proof. Assume that

$$
\mu=\sum_{i=1}^{k} a_{i} \mu_{i},
$$

where $\mu_{i} \in \hat{\Lambda}, a_{i} \in \mathbb{R}, a_{i}>0, k>1$ and $\sum_{i=1}^{k} a_{i}=1$. We may even assume that $k$ is minimal possible. The latter says that $\mu$ belongs to the interior of the convex hull $H$ of the $\mu_{i}$ 's. By [La, Corollary 2.7.2] we may assume that $\mu_{i}-\mu, i=1, \ldots, k$, are affinely independent.

Denote by $X$ the subspace of $\mathbb{R}^{n}$, generated by the elements $\mu_{i}-\mu$, $i=1, \ldots, k$. Then $H-\mu \subset X$ and since $\mu$ is a point in the interior of $H$, we get that the convex cone in $X$ with origin in $\mu$, which contains all $\mu_{i}-\mu, i=1, \ldots, k$, coincides with $X$. By [La, Lemma 2.6.2] we have $\operatorname{dim} X=k-1$ and hence without loss of generality we may assume that the elements $\mu_{i}-\mu, i=2, \ldots, k$, are linearly independent. Then from (4.1) we have

$$
-\left(\mu_{1}-\mu\right)=\sum_{j=2}^{k} \frac{a_{i}}{a_{1}}\left(\mu_{i}-\mu\right) .
$$

As the vectors $\mu_{i}-\mu, i=2, \ldots, k$, are linearly independent, the equation (4.2) gives the unique linear combination of these elements, which is equal to $-\left(\mu_{1}-\mu\right)$. Since all involved elements $\mu_{i}, \mu$ are from $\mathbb{Z}^{n}$, it follows that all $\frac{a_{i}}{a_{1}}$ in (4.2) are rational numbers (and are positive). Multiplying, if necessary, with the denominator, we obtain the equality

$$
-b_{1}\left(\mu_{1}-\mu\right)=\sum_{j=2}^{k} b_{i}\left(\mu_{i}-\mu\right),
$$

where all $b_{i}$ 's are positive integers. 
Note that for every $i=1,2, \ldots, k$ we have $\mathfrak{g}_{\mu_{i}-\mu} V_{\mu} \subset V_{\mu_{i}}=0$ by our assumptions. From (4.3) we see that $\mathfrak{g}_{0}$ is in the Lie subalgebra generated by all $\mathfrak{g}_{\mu_{i}-\mu}, i=1,2, \ldots, k$. Therefore $\mathfrak{g}_{0} V_{\mu}=0$. This implies $\mu=0$ or $V_{\mu}=0$ and completes the proof of our lemma.

Let $\bar{\Lambda}$ denote the convex hull of $\hat{\Lambda}$ under the usual topology in $\mathbb{R}^{n}$. As the module $V$ is not dense, we have $\bar{\Lambda} \neq \varnothing$. As the module $V$ is not trivial, it must contain a nonzero weight and hence $\bar{\Lambda} \neq \mathbb{R}^{n}$ by Lemma 9. From the hyperplane separation theorem (see e.g. [La, 3.2]) it follows that for every $\mu \in \operatorname{supp}(V) \backslash\{0\}$ there exists $a_{\mu} \in \mathbb{R}^{n}, a_{\mu} \neq 0$, such that $\hat{\Lambda} \subset \mu+\mathbb{Z}_{+}^{\left(a_{\mu}\right)}$. Thus for the fix $\lambda$ there is a nonzero $a \in \mathbb{R}^{n}$ such that $\hat{\Lambda} \subset \lambda+\mathbb{Z}_{+}^{(a)}$.

To proceed we will need a simple fact about ordered abelian groups.

Lemma 10. Let $\beta \in \mathbb{Z}^{n}$ be such that $\beta \cdot a<0$. Then there exists a finite collection of elements $\beta_{1}^{ \pm}, \ldots, \beta_{n}^{ \pm}$from $\mathbb{Z}_{+}^{(a)}$ such that $\left\{\beta, \beta_{1}^{ \pm}, \ldots, \beta_{n}^{ \pm}\right\}$ generates $\mathbb{Z}^{n}$ as a semigroup.

Proof. As $\beta \cdot a<0$, for every $\alpha \in \mathbb{Z}^{n}$ we can fix some $k_{\alpha} \in\{0,1, \ldots\}$ such that $\alpha-k_{\alpha} \beta \in \mathbb{Z}_{+}^{(a)}$. For $i \in\{1, \ldots, n\}$ set $\beta_{i}^{ \pm}= \pm e_{i}-k_{ \pm e_{i}} \beta$. Then all elements $\pm e_{i}$ belong to the semigroup, generated by $\left\{\beta, \beta_{1}^{ \pm}, \ldots, \beta_{n}^{ \pm}\right\}$, by construction and hence the latter set generates the whole $\mathbb{Z}^{n}$ as a semigroup.

For every $\beta \in \mathbb{Z}^{n}$ with $\beta \cdot a<0$, we fix $\beta_{1}^{ \pm}, \ldots, \beta_{n}^{ \pm}$given by Lemma 10 . Now we can generalize our analysis from Section 3 .

Lemma 11. Let $\beta \in \mathbb{Z}^{n}$ be such that $\beta \cdot a<0$. Then there exists $\mu \in \operatorname{supp}(V)$ such that $\mu-\beta \in \hat{\Lambda}$.

Proof. Let $\nu \in \hat{\Lambda} \neq \varnothing$. Consider the ray $\{\nu+k \beta: k \in \mathbb{N}\}$. As $\beta \cdot a<0$, this ray must intersect $\lambda+\mathbb{Z}_{-}^{(a)} \subset \operatorname{supp}(V)$. The claim follows.

Lemma 12. Let $v \in V$ be a nonzero weight vector. Assume that there exists $\beta \in \mathbb{Z}^{n}$ such that $\beta \cdot a<0$ and $\mathfrak{g}_{\beta} v=0$. Then for any $w \in V$ there exists $N \in \mathbb{N}$ such that for any $\alpha=b \beta+\gamma$, where $b \in \mathbb{N}$ and $\gamma \in \mathbb{N}^{n}$ with $\gamma>(N, N, \ldots, N)$, we have $\mathfrak{g}_{\alpha} w=0$.

Proof. From Lemma 3 we know that $v$ is a generalized highest weight element and hence there exists $N_{1} \in \mathbb{N}$ such that we have $\mathfrak{g}_{\gamma} v=0$ for all $\gamma \in \mathbb{N}^{n}, \gamma>\left(N_{1}, N_{1}, \ldots, N_{1}\right)$. If not all components $\beta_{i}$ of $\beta$ are negative, using that $\left[\mathfrak{g}_{x}, \mathfrak{g}_{y}\right]=\mathfrak{g}_{x+y}$ if $x \neq y$, in the case $w=v$ the claim of the lemma follows by induction on $b$ and taking $N=N_{1}$. Now suppose all components $\beta_{i}$ of $\beta$ are negative. In the case $w=v$ we take $N=2 N_{1}+2$. For $\gamma>(N, N, \ldots, N)$ with $0 \notin \mathbb{N} \beta+\gamma$ the claim of the lemma follows by induction on $b$. For $\gamma>(N, N, \ldots, N)$ with $\gamma=-k \beta, k>0$, we write $\gamma=\gamma_{1}+\gamma_{2}$ such that $\gamma_{1}, \gamma_{2}>(N, N, \ldots, N)$, $0 \notin \mathbb{N} \beta+\gamma_{1}$ and $\gamma_{2} \neq b \beta+\gamma_{1}$. Then we know that $\mathfrak{g}_{b \beta+\gamma_{1}} v=0$, further $\mathfrak{g}_{b \beta+\gamma} v=0$. The claim in the Lemma follows for the case $w=v$. 
Using the same arguments as in Lemma 3 one shows that if the claim of our lemma is true for some $w$, it is true for any element from $\mathfrak{g}_{x} w$, $x \in \mathbb{Z}^{n}$. The general claim of the lemma now follows from the fact that $V$ is simple and hence generated by $v$.

Lemma 13. Let $v \in V$ be a nonzero weight vector. Assume that there exists $\beta \in \mathbb{Z}^{n}$ such that $\beta \cdot a<0$ and $\mathfrak{g}_{\beta} v=0$. Then there exists $N \in \mathbb{N}$ such that for any $\alpha=b \beta+\sum_{\varepsilon \in\{ \pm\}} \sum_{i=1}^{n} b_{i}^{\varepsilon} \beta_{i}^{\varepsilon}+\gamma$, where $b, b_{i}^{ \pm} \in \mathbb{N}$, $i=1, \ldots, n$, and $\gamma \in \mathbb{N}^{n}$ with $\gamma>(N, N, \ldots, N)$, we have $\mathfrak{g}_{\alpha} v=0$.

Proof. By Lemma 11 for every $\varepsilon \in\{ \pm\}$ and $i \in\{1,2, \ldots, n\}$ there is $\mu_{i}^{\varepsilon} \in \operatorname{supp}(V)$ such that $\mu_{i}^{\varepsilon}+\beta_{i}^{\varepsilon} \in \hat{\Lambda}$. This yields $\mathfrak{g}_{\beta_{i}^{\varepsilon}} V_{\mu_{i}^{\varepsilon}}=0$. Hence, by Lemma 12, there exists $N_{i}^{\varepsilon} \in \mathbb{N}$ such that

$$
\mathfrak{g}_{b_{i}^{\varepsilon} \beta_{i}^{\varepsilon}+\gamma_{i}^{\varepsilon}} v=0
$$

for any $b_{i}^{\varepsilon} \in \mathbb{N}$ and any $\gamma_{i}^{\varepsilon} \in \mathbb{N}^{n}, \gamma_{i}^{\varepsilon}>\left(N_{i}^{\varepsilon}, N_{i}^{\varepsilon}, \ldots, N_{i}^{\varepsilon}\right)$.

Similarly, there exists $N_{\beta} \in \mathbb{N}$ such that

$$
\mathfrak{g}_{b \beta+\gamma^{\prime}} v=0
$$

for any $b \in \mathbb{N}$ and any $\gamma^{\prime} \in \mathbb{N}^{n}, \gamma^{\prime}>\left(N_{\beta}, N_{\beta}, \ldots, N_{\beta}\right)$. Let

$$
N=N_{\beta}+\sum_{\varepsilon \in\{ \pm\}} \sum_{i=1}^{n} N_{i}^{\varepsilon}+2 n+1 \text {. }
$$

Then every $\gamma>(N, N, \ldots, N)$ can be written as

$$
\gamma=\gamma^{\prime}+\sum_{\varepsilon \in\{ \pm\}} \sum_{i=1}^{n} \gamma_{i}^{\varepsilon}
$$

where $\gamma^{\prime}>\left(N_{\beta}, N_{\beta}, \ldots, N_{\beta}\right)$ and $\gamma_{i}^{\varepsilon}>\left(N_{i}^{\varepsilon}, N_{i}^{\varepsilon}, \ldots, N_{i}^{\varepsilon}\right)$. For this choice of $N$ using the expression

$$
b \beta+\sum_{\varepsilon \in\{ \pm\}} \sum_{i=1}^{n} b_{i}^{\varepsilon} \beta_{i}^{\varepsilon}+\gamma=\left(b \beta+\gamma^{\prime}\right)+\sum_{\varepsilon \in\{ \pm\}} \sum_{i=1}^{n}\left(b_{i}^{\varepsilon} \beta_{i}^{\varepsilon}+\gamma_{i}^{\varepsilon}\right),
$$

we deduce the claim of the lemma from (4.4) and (4.5).

Lemma 14. Let $\mu \in \hat{\Lambda}$ and $\beta \in \mathbb{Z}^{n}$ be such that $\beta \cdot a<0$. Then $\mu-\beta \in \hat{\Lambda}$.

Proof. We have $\mathfrak{g}_{\beta} V_{\mu-\beta} \subset V_{\mu}=0$. Suppose $V_{\mu-\beta} \neq 0$ and fix any nonzero $v \in V_{\mu-\beta}$. By Lemma 13, there exists $N \in \mathbb{N}$ such that for any $\alpha=b \beta+\sum_{i=1}^{n} b_{i}^{ \pm} \beta_{i}^{ \pm}+\gamma$, where $b, b_{i}^{ \pm} \in \mathbb{N}, i=1, \ldots, n$, and $\gamma \in \mathbb{N}^{n}$ with $\gamma>(N, N, \ldots, N)$, we have $\mathfrak{g}_{\alpha} v=0$.

Since $\left\{\beta, \beta_{1}^{ \pm}, \ldots, \beta_{n}^{ \pm}\right\}$generate $\mathbb{Z}^{n}$ as a semigroup (Lemma 10), for any $\alpha \in \mathbb{Z}^{n}$ we can write

$$
\alpha-(N+1, N+1, \ldots, N+1)=b \beta+\sum_{\varepsilon \in\{ \pm\}} \sum_{i=1}^{n} b_{i}^{\varepsilon} \beta_{i}^{ \pm}
$$


for some $b, b_{i}^{ \pm} \in \mathbb{N}$ and hence

$$
\alpha=b \beta+\sum_{\varepsilon \in\{ \pm\}} \sum_{i=1}^{n} b_{i}^{\varepsilon} \beta_{i}^{ \pm}+(N+1, N+1, \ldots, N+1) .
$$

Note that $(N+1, N+1, \ldots, N+1)>(N, N, \ldots, N)$, which yields $\mathfrak{g}_{\alpha} v=0$ from the previous paragraph. Thus $V$ is a trivial module which is a contradiction. Therefore $V_{\mu-\beta}=0$ which completes the proof.

Theorem 8 follows directly from Lemma 14.

4.2. Case $n=2$. In the case $n=2$ Theorem 8 implies the following trichotomy result for all weight modules (see [Ma2]):

Corollary 15. Assume that $n=2$ and $V$ is a simple weight $\mathfrak{g}$-module. Then $V$ is either dense or punctured or cut.

Proof. We work with the same setup (for $\Lambda$ and $\hat{\Lambda}$ ) as in Theorem 8 . Note that the trivial $\mathfrak{g}$-module is obviously cut. Hence, because of Theorem 8, it is enough to show that any simple nontrivial weight module $V$ for which $|\hat{\Lambda}|>1$ contains a generalized highest weight element.

Now suppose $|\hat{\Lambda}|>1$. If $\hat{\Lambda}$ contains a line in $\mathbb{Z}^{n}$, we can easily see that $V$ is cut (we are using the identification defined before Lemma 9). Now we also suppose that each line in $\mathbb{Z}^{n}$ has at least one weight of $V$. By changing the coordinate system $\left\{e_{1}, e_{2}\right\}$ and changing $\lambda$ (which is allowed to be 0$)$ if necessary, we may assume that $(0,0) \notin \hat{\Lambda}$ and $(1,0),(1, k) \in \hat{\Lambda}$ for some $k \in \mathbb{N}$. Using Lemma 9 we may even assume $k \in\{1,2\}$. If $(1,1) \in \operatorname{supp}(V)$ we must have $\lambda+e_{1}+e_{2}=0$, and further $\hat{\Lambda}=\{(1,0),(1,2)\}$ (otherwise we use other points instead of $(1,0),(1,2)$ to get $k=1)$.

If $(1,1) \in \hat{\Lambda}$, then $\mathfrak{g}_{e_{1}} V_{\lambda}=\mathfrak{g}_{e_{1}+e_{2}} V_{\lambda}=0$ and since $\left\{e_{1}, e_{1}+e_{2}\right\}$ is a $\mathbb{Z}$-basis of $\mathbb{Z}^{2}$ we obtain that any element in $V_{\lambda}$ is a generalized highest weight element.

Finally, we are left with the case $\hat{\Lambda}=\{(1,0),(1,2)\}$ (and we also have the equality $\lambda+e_{1}+e_{2}=0$, which will not be used). Consider the $\mathbb{Z}$-basis $\alpha=e_{1}, \beta=2 e_{1}+e_{2}$ of $\mathbb{Z}^{2}$. We claim that in this case for any $a, b \in \mathbb{N}$ such that $a, b>1$ we have $\mathfrak{g}_{a \alpha+b \beta} V_{\lambda}=0$ (and hence any element in $V_{\lambda}$ is a generalized highest weight element with respect to the basis $\{\alpha+\beta, \alpha+2 \beta\})$. As $\mathfrak{g}_{\alpha} V_{\lambda}=0$ by our assumptions, by induction it is enough to show that $\mathfrak{g}_{b \beta} V_{\lambda}=0$ for all $b \in \mathbb{N}, b>1$.

If $b$ is even we have $b \beta=\frac{b}{2}\left(e_{1}+2 e_{2}\right)+\frac{3 b}{2} e_{1}$ and $\mathfrak{g}_{b \beta} V_{\lambda}=0$ follows from $\mathfrak{g}_{e_{1}+2 e_{2}} V_{\lambda} \subset V_{\lambda+e_{1}+2 e_{2}}=0$ and $\mathfrak{g}_{e_{1}} V_{\lambda} \subset V_{\lambda+e_{1}}=0$.

If $b=2 k+1$ is odd (in particular, $b \geqslant 3$ ), we write $b \beta=\left(b \beta-e_{2}\right)+e_{2}$ and have

$$
\mathfrak{g}_{b \beta} V_{\lambda}=\left[\mathfrak{g}_{b \beta-e_{2}}, \mathfrak{g}_{e_{2}}\right] V_{\lambda} \subset \mathfrak{g}_{b \beta-e_{2}} V_{\lambda+e_{2}}+\mathfrak{g}_{e_{2}} \mathfrak{g}_{b \beta-e_{2}} V_{\lambda}
$$


Similarly to the argument in the previous paragraph we have $\mathfrak{g}_{b \beta-e_{2}} V_{\lambda}=$ 0 . Note that $\mathfrak{g}_{e_{1}+e_{2}} V_{\lambda+e_{2}} \subset V_{\lambda+e_{1}+2 e_{2}}=0, \mathfrak{g}_{e_{1}-e_{2}} V_{\lambda+e_{2}} \subset V_{\lambda+e_{1}}=0$ and that $b \beta-e_{2}=(4 k+2) e_{1}+2 k e_{2}=(3 k+1)\left(e_{1}+e_{2}\right)+(k+1)\left(e_{2}-e_{2}\right)$. Then we have $\mathfrak{g}_{b \beta-e_{2}} V_{\lambda+e_{2}}=0$, and further $\mathfrak{g}_{b \beta} V_{\lambda}=0$. This completes the proof.

4.3. Mixed modules. Another way to generalize the results of this paper is to study the supports of the so-called mixed modules. A weight $\mathfrak{g}$-module $V$ is called mixed (see [Ma4]) provided that there exists $\lambda \in \operatorname{supp}(V)$ and $\alpha \in \mathbb{Z}^{n}$ such that $\operatorname{dim} V_{\lambda}=\infty$ and $\operatorname{dim} V_{\lambda+\alpha}<\infty$. In [MZ] it is shown that for the Virasoro algebra mixed modules do not exist. However, for $n>1$ simple highest weight $W_{n}$-modules are mixed in the general case (this follows for example from [HWZ]). Hence it is natural to ask whether there are other classes of simple mixed modules, for example if there are mixed dense or mixed punctured modules.

Conjecture 16. Any mixed $\mathfrak{g}$-module is cut.

Below we give some motivation and (weak) evidence for this conjecture. Denote by $\operatorname{supp}^{\infty}(V)$ the set of all $\lambda \in \mathfrak{g}_{0}^{*} \operatorname{such}$ that $\operatorname{dim} V_{\lambda}=\infty$. For a simple weight $\mathfrak{g}$-module $V$ we also denote by $\operatorname{supp}^{\mathrm{fin}}(V)$ the set of all $\lambda \in \operatorname{supp}(V)+\mathbb{Z}^{n}$ such that $\operatorname{dim} V_{\lambda}<\infty$. Note that $\operatorname{supp}^{\mathrm{fin}}(V)$ may not be a subset of $\operatorname{supp}(V)$. Let $\Lambda=\operatorname{supp}(V)+\mathbb{Z}^{n}$.

Lemma 17. Let $V$ be a simple mixed module and $\mu \in \Lambda$ be a convex linear combination of some elements from $\operatorname{supp}^{\text {fin }}(V)$. Then we have either $\mu=0$ or $\mu \in \operatorname{supp}^{\text {fin }}(V)$.

Proof. Let $\mu$ be a convex linear combination of some $\mu_{1}, \ldots, \mu_{k} \in$ $\operatorname{supp}^{\mathrm{fin}}(V)$ as in the proof of Lemma 9. Assume $\mu \in \operatorname{supp}^{\infty}(V)$. Then $\operatorname{dim} V_{\mu}=\infty$ while $\operatorname{dim} V_{\mu_{i}}<\infty$ for all $i=1, \ldots, k$. Hence there exists $v \in V_{\mu}, v \neq 0$, such that $\mathfrak{g}_{\mu_{i}-\mu} v=0$ for all $i=1, \ldots, k$. Repeating the arguments from the proof of Lemma 9 we obtain $\mathfrak{g}_{0} v=0$, which implies $\mu=0$. The claim follows.

Corollary 18. Any simple mixed module $V$ containing a generalized highest weight element is a cut module.

Proof. We may assume that every element in $V$ is a generalized highest weight element with respect to the basis $e_{1}, \ldots, e_{n}$. Let $\lambda \in \operatorname{supp}^{\text {fin }}(V)$. Then for any $\alpha \in \mathbb{N}^{n}$ we have $\lambda+\alpha \in \operatorname{supp}^{\text {fin }}(V)$ for otherwise $\operatorname{dim} V_{\lambda+\alpha}=\infty$ and hence $V_{\lambda+\alpha}$ must contain a nonzero vector $v$, annihilated by $\mathfrak{g}_{-\alpha}$. Using that $v$ is a generalized highest weight element and $\mathfrak{g}_{-\alpha} v=0$ one shows that $\mathfrak{g} v=0$, implying that $V$ is trivial and hence not mixed, a contradiction.

From $\lambda+\alpha \in \operatorname{supp}^{\text {fin }}(V)$ for any $\alpha \in \mathbb{N}^{n}$, similarly to the proof of Lemma 5 one shows that $V$ is not dense. Hence the claim follows from Theorem 8. 
Corollary 19. Let $n=2$. Then any simple mixed module $V$ for which $\left|\operatorname{supp}^{\operatorname{fin}}(V)\right|>1$ is a cut module.

Proof. Using Lemma 17, similarly to the proof of Corollary 15 we may assume $\lambda \in \operatorname{supp}^{\infty}(V), \lambda+e_{1} \in \operatorname{supp}^{\text {fin }}(V)$ and either $\lambda+e_{1}+e_{2} \in$ $\operatorname{supp}^{\mathrm{fin}}(V)$ or $\lambda+e_{1}+2 e_{2} \in \operatorname{supp}^{\mathrm{fin}}(V)$.

In the first case from $\operatorname{dim} V_{\lambda}=\infty$ and $\operatorname{dim} V_{\lambda+e_{1}}, \operatorname{dim} V_{\lambda+e_{1}+e_{2}}<\infty$ we have that there exists $v \in V_{\lambda}$, annihilated by both $\mathfrak{g}_{e_{1}}$ and $\mathfrak{g}_{e_{1}+e_{2}}$. As $\left\{e_{1}, e_{1}+e_{2}\right\}$ is a $\mathbb{Z}$-basis of $\mathbb{Z}^{2}$, the element $v$ is a generalized highest weight element and the claim follows from Corollary 18.

In the second case $\left(\lambda+e_{1}+2 e_{2} \in \operatorname{supp}^{\text {fin }}(V)\right)$ one proves the existence of a generalized highest weight element in $V_{\lambda}$ similarly to the proof of Corollary 18. The claim follows.

Corollary 20. Let $n=2$. Then for any simple mixed punctured module $V$ we have $\operatorname{supp}^{\infty}(V)=\operatorname{supp}(V)$.

Proof. This follows from Corollary 19 and definitions.

\section{Acknowledgments}

The research was done during the visit of the first author to Wilfrid Laurier University in April and May 2009. The hospitality and financial support of Wilfrid Laurier University are gratefully acknowledged. The first author was partially supported by the Swedish Research Council. The second author was partially supported by NSERC and NSF of China (Grant 10871192). We thank Svante Janson for helpful discussions.

\section{REFERENCES}

[BZ] $Y$. Billig and K. Zhao Weight modules over exp-polynomial Lie algebras. J. Pure Appl. Algebra 191 (2004), no. 1-2, 23-42.

[DMP] I. Dimitrov, O. Mathieu, I. Penkov, On the structure of weight modules. Trans. Amer. Math. Soc. 352 (2000), no. 6, 2857-2869.

[ER] S. Eswara Rao, Irreducible representations of the Lie-algebra of the diffeomorphisms of a $d$-dimensional torus. J. Algebra 182 (1996), no. 2, 401-421.

[Fe] S. Fernando, Lie algebra modules with finite-dimensional weight spaces. I. Trans. Amer. Math. Soc. 322 (1990), no. 2, 757-781.

[Fu] V. Futorny, Weight representations of semi-simple finite-dimensional Lie algebras, Ph.D. Thesis, Kyiv University, Kyiv, Ukraine, 1986.

[Ga] P. Gabriel, Lectures at the Seminaire Godement, Paris, unpublished notes, 1959.

[HWZ] J. Hu, X. Wang, K. Zhao, Verma modules over generalized Virasoro algebras Vir $[G]$. J. Pure Appl. Algebra 177 (2003), no. 1, 61-69.

[La] $N$. Lauritzen, Lectures on convex sets, notes available online from: http://home.imf.au.dk/niels/lecconset.pdf

[LZ] R. Lu and K. Zhao, Classification of irreducible weight modules over higher rank Virasoro algebras, Adv. Math. 201 (2006), no. 2, 630-656.

[M1] O. Mathieu, Classification of Harish-Chandra modules over the Virasoro Lie algebra. Invent. Math. 107 (1992), no. 2, 225-234. 
[M2] O. Mathieu, Classification of irreducible weight modules. Ann. Inst. Fourier (Grenoble) 50 (2000), no. 2, 537-592.

[Ma1] V. Mazorchuk, Futorny theorem for generalized Witt algebras of rank 2. Comm. Algebra 25 (1997), no. 2, 533-541.

[Ma2] V. Mazorchuk, On the support of irreducible modules over the WittKaplansky algebras of rank (2, 2). Mathematika 45 (1998), no. 2, 381-389.

[Ma3] V. Mazorchuk, Classification of simple Harish-Chandra modules over QVirasoro algebra. Math. Nachr. 209 (2000), 171-177.

[Ma4] V. Mazorchuk, On simple mixed modules over the Virasoro algebra. Mat. Stud. 22 (2004), no. 2, 121-128.

[Ma5] V. Mazorchuk, Lectures on $\mathfrak{s l}_{2}(\mathbb{C})$-modules, to be published by Imperial College Press.

[MZ] V. Mazorchuk and K. Zhao, Classification of simple weight Virasoro modules with a finite-dimensional weight space. J. Algebra 307 (2007), no. 1, 209-214.

[PZ] J. Patera and H. Zassenhaus, The higher rank Virasoro algebras, Comm. Math. Phys. 136 (1991), 1-14.

[PS] I. Penkov, V. Serganova, Weight representations of the polynomial Cartan type Lie algebras $W_{n}$ and $\bar{S}_{n}$. Math. Res. Lett. 6 (1999), no. 3-4, 397-416.

[Sh] G. Shen, Graded modules of graded Lie algebras of Cartan type. I. Mixed products of modules. Sci. Sinica Ser. A 29 (1986), no. 6, 570-581.

[Su] $\quad Y . S u$, Simple modules over the high rank Virasoro algebras. Comm. Algebra 29 (2001), no. 5, 2067-2080.

V.M.: Department of Mathematics, Uppsala University, Box 480, SE751 06, Uppsala, SWEDEN; e-mail: mazor@math.uu.se

K.Z.: Department of Mathematics, Wilfrid Laurier University, Waterloo, Ontario, N2L 3C5, Canada; and Institute of Mathematics, Academy of Mathematics and System Sciences, Chinese Academy of Sciences, Beijing 100190, PR China; e-mail: kzhao@wlu.ca 